\title{
Erratum: Splenomegaly in Colon Cancer During Adjuvant Oxaliplatin-based Chemotherapy
}

\author{
Tulay Eren ${ }^{1}$, Lale Pasaoglu ${ }^{2}$ \\ 1. Oncology, Diskapi Yildirim Beyazit Training and Research Hospital, Ankara, TUR 2. Radiology, Ankara \\ City Hospital, Ankara, TUR
}

Corresponding author: Tulay Eren, tulayeren78@gmail.com

Corresponding author: Tulay Eren

1. Oncology, Diskapi Yildirim Beyazit Training and Research Hospital, Ankara, TUR 2. Radiology, Ankara City Hospital, Ankara, TUR

How to cite this erratum

Eren T, Pasaoglu L (June 17, 2020) Correction: Splenomegaly in Colon Cancer During Adjuvant Oxaliplatin-based Chemotherapy. Cureus 12(6): c32. doi:10.7759/cureus.c32

\section{Correction Notice}

A decimal point was erroneously included in the initial manuscript submission. This error was, regrettably, not caught during peer or editorial review. As a result, the decimal point has been removed from " $94.4 \mathrm{mg} / \mathrm{m} 2$ " as seen in the following sentence located in the results section: "The optimum cut-off value for age was 60 years, sensitivity $60 \%$ and specificity $56 \%$; the same values for cumulative oxaliplatin dosage were, respectively, $\mathbf{9 4 . 4} \mathbf{m g} / \mathrm{m} 2$, 72\%, and 68\%."

The corrected sentence now reads: "The optimum cut-off value for age was 60 years, sensitivity $60 \%$ and specificity $56 \%$; the same values for cumulative oxaliplatin dosage were, respectively, $944 \mathrm{mg} / \mathrm{m} 2,72 \%$, and 68\%."

Cureus sincerely regrets this error. 